\title{
ECONOMETRIC METHODS FOR IMPROVED MEASURES \\ OF FINANCIAL RISK
}

\author{
Moohwan Kim \\ Dr. Douglas J. Miller, Dissertation Supervisor
}

\begin{abstract}
Faced with the current financial crisis, several US and foreign banks and investment firms have failed due to excessive losses. The Value-at-Risk (VaR) was a widely-used risk model that was problematic. We evaluate competing claims from the financial economics literature about the relative importance of the VaR flaws (e.g., subadditivity) and probability model specification errors in risk measurement under Extreme Value Theory. In particular, we use the peaks-over-threshold method based on the Generalized Pareto model to compare the relative performance of the VaR and Conditional VaR for assessing forward-looking risk in observed hedge fund returns. In the second chapter, focus is on how to improve our ability to manage financial risks by developing a better understanding of the microstructure of financial markets. Using high frequency foreign exchange rate data, we want to see if we can better assess the current risk of financial positions and improve our predictions of future price movements. To handle the fact that high frequency returns might be correlated in a nonlinear fashion, we may use copula-based probability models that are involved with GARCH models. We compare the performance of the alternative dynamic hedging models with the hedging effectiveness of the static model. In chapter three, we try to choose an appropriate copula model that provides the "best" fit to the observed data. Along with the discussions of the existing model selection procedures, we propose a non-nested test procedure for copula model selection that is based on the Cox test statistic.
\end{abstract}

